\title{
A MAXIMIN APPROACH FOR ROBUST MIMO DESIGN: COMBINING OSTBC AND BEAMFORMING WITH MINIMUM TRANSMISSION POWER REQUIREMENTS
}

\author{
Antonio Pascual-Iserte, Ana I. Pérez-Neira, Miguel Ángel Lagunas \\ Department of Signal Theory and Communications Telecommunications Technological Center of Catalonia \\ Polytechnic University of Catalonia (UPC) CTTC - Edifici NEXUS I \\ C/ Jordi Girona 1-3 (Campus Nord UPC - mòdul D5), 08034 Barcelona (SPAIN) \\ e-mail:\{tonip,anuska\}@gps.tsc.upc.es, m.a.lagunas@cttc.es
}

\begin{abstract}
In this paper we address the problem of the design of a transmitter in a Multi-Input-Multi-Output (MIMO) space diversity system. We consider a design which is robust to errors in the channel estimate available at the transmitter. The design is based on the minimization of the necessary transmit power while guaranteeing a minimum and specified Quality of Service (QoS) in terms of Signal to Noise Ratio (SNR) with a certain probability. According to this objective, the transmitter exploits all the eigenmodes of the MIMO channel estimate, distributing the transmit power in a robust way among the channel modes by using orthonormal temporal signatures. In order to guarantee a full rate transmission, the orthonormal temporal signatures designed for real constellations in Orthogonal Space Time Block Codes (OSTBC) are used, leading to a system structure based on the combination of OSTBC and orthogonal weighted beamforming.
\end{abstract}

\section{INTRODUCTION}

In last years, much attention has been paid to the use of antenna arrays to improve the quality and the rate of transmission and to cope with the impairments produced by the scatterer wireless channel. There exist two different groups of techniques, those based on space-time codes when no Channel State Information (CSI) is available at the transmitter, such as [1] and [2], among others, and those based on linear pre-processing or beamforming when the transmitter knows the channel response ([3] [4]). In a realistic deployment, only a channel estimate is available, and therefore, the already proposed techniques should be redefined in order to take into account the errors in the estimate, leading to the so called robust techniques.

Most of the already known robust designs are based on a statistical or Bayesian approach, in which the mean value of a goodness function averaged over the statistics of the real channel conditioned to the channel estimate is maximized ([5] [6]). One feature of this kind of techniques is that, although they optimize the mean behaviour of the system, no minimum quality of service can be guaranteed to the user. In a packet transmission network, this parameter has a special relevance, due to the fact that a packet has to

This work has been partially financed by the Spanish Government under projects GIRAFA TIC2002-04594-C02 (jointly financed by FEDER), MARQUIS FIT-070000-2003-257 (MEDEA+ A111), and the integrated action HF2001-0055; and by the European Union under project WINNER IST-2003-507581. be accepted or disregarded. Obviously, a technique able to establish a relationship between a minimum quality of transmission for accepting a packet and the associated probability is desired.

In this paper it is proposed a robust design that takes into account explicitly the presence of errors in the Multi-Input-MultiOutput (MIMO) channel estimate available at the transmitter. All the eigenmodes of the channel estimate are used, and the available power is distributed in a robust way among these modes according to a criterion that maximizes the Signal to Noise Ratio (SNR) for the worst real channel that can be found given the channel estimate, that is, a maximin robust approach [7] is taken instead of the classical Bayesian philosophy. Other important worst-case optimization problems have been solved previously, such as in [8].

This paper is organized as follows. In Section 2 the system and signal models are presented. Sections 3 and 4 present the nonrobust and the maximin robust designs, respectively. Finally, in Section 5 some simulation results and conclusions are shown.

\section{SYSTEM AND SIGNAL MODELS}

We consider the transmission through a flat fading spatially uncorrelated Rayleigh MIMO channel with $n_{T}$ transmit and $n_{R}$ receive antennas. The $(i, j)$ th component in the matrix $\mathbf{H} \in \mathbb{C}^{n_{R} \times n_{T}}$ represents the gain factor between the $j$ th transmit and the $i$ th receive antenna. All the components in the MIMO channel matrix are i.i.d. complex and circularly symmetric Gaussian variables with zero mean and variance $\sigma_{h}^{2}$. At the transmitter only a channel estimate $\hat{\mathbf{H}}$ is available, corresponding to the following model: $\widehat{\mathbf{H}}=\mathbf{H}+\mathbf{E}$, where $\mathbf{E}$ represents the error in the channel estimate, and is composed by i.i.d. complex and circularly symmetric Gaussian variables with variance $\sigma_{e}^{2}$ and independent from $\mathbf{H}$. According to this distribution, it is easy to verify that the real channel conditioned to the channel estimate follows a Gaussian law:

$$
\begin{aligned}
\mathbf{h} & =\operatorname{vec}(\mathbf{H}), \quad \widehat{\mathbf{h}}=\operatorname{vec}(\widehat{\mathbf{H}}) \\
f(\mathbf{h} \mid \widehat{\mathbf{h}}) & \sim G(\mathbf{m}, \mathbf{C}), \quad \mathbf{m}=\frac{\sigma_{h}^{2}}{\sigma_{h}^{2}+\sigma_{e}^{2}} \widehat{\mathbf{h}}=\frac{\mathrm{SNR}_{\mathrm{est}}}{1+\mathrm{SNR}_{\mathrm{est}}} \widehat{\mathbf{h}} \\
\mathbf{C} & =\frac{\sigma_{h}^{2} \sigma_{e}^{2}}{\sigma_{h}^{2}+\sigma_{e}^{2}} \mathbf{I}=\frac{\sigma_{h}^{2}}{1+\mathrm{SNR}_{\mathrm{est}}} \mathbf{I}, \quad \mathrm{SNR}_{\mathrm{est}}=\frac{\sigma_{h}^{2}}{\sigma_{e}^{2}}
\end{aligned}
$$

where $\operatorname{vec}(\cdot)$ represents the operator that stacks columnwise all the columns of a matrix, and $\mathbf{m}$ is the Minimum Mean Square Error (MMSE) Bayesian estimate of the channel. $\widehat{\mathbf{H}}_{b}$ represents this Bayesian channel estimate, i.e., $\widehat{\mathbf{H}}_{b}=\frac{\mathrm{SNR}_{\mathrm{est}}}{1+\mathrm{SNR}_{\mathrm{est}}} \widehat{\mathbf{H}}$. 
The transmission scheme is based on a matrix modulation. That means, that if a symbol $s(n)$ has to be transmitted, then the following signal is transmitted $\mathbf{X}_{T}(n)=\mathbf{B} s(n)$, where the matrix $\mathbf{B} \in \mathbb{C}^{n_{T} \times n_{T}}$ contains the factors that multiply the symbol $s(n)$ before the signal is transmitted through the $n_{T}$ transmit antennas during $n_{T}$ channel uses or periods of time. The transmit power constraint can be formulated in terms of the matrix $\mathbf{B}$ as $\|\mathbf{B}\|_{F}^{2}=\operatorname{tr}\left(\mathbf{B}^{H} \mathbf{B}\right)=P_{T}$. According to this matrix modulation scheme, the received samples at all the receive antennas during the $n_{T}$ periods of time corresponding to the transmission of $s(n)$ can be expressed as: $\mathbf{X}(n)=\mathbf{H B} s(n)+\mathbf{W}(n) \in \mathbb{C}^{n_{R} \times n_{T}}$, where $\mathbf{W}(n)$ models the AWGN contribution, with variance $\sigma_{w}^{2}$.

We assume that the receiver knows perfectly which is the transmitter, i.e., the matrix $\mathbf{B}$, and the channel realization $\mathbf{H}$. According to this, the optimum receiver is based on the matched filter, which extracts the following sufficient statistic $d(n)$ in order to carry out the detection: $d(n)=\operatorname{tr}\left(\mathbf{A}^{H} \mathbf{X}\right)$, where $\mathbf{A}=\mathbf{H B}$, so that $d(n)=\operatorname{tr}\left(\mathbf{B}^{H} \mathbf{H}^{H} \mathbf{H B}\right) s(n)+\operatorname{tr}\left(\mathbf{B}^{H} \mathbf{H}^{H} \mathbf{W}(n)\right)$. The SNR is maximized by using this receiver, leading to the following expression: SNR $=\frac{\sigma_{s}^{2}}{\sigma_{w}^{2}} \operatorname{tr}\left(\mathbf{B}^{H} \mathbf{H}^{H} \mathbf{H B}\right)$. In this paper we force the matrix $\mathbf{B}$ to have the following structure, which is able to accommodate several transmission architectures, as it will be shown in other sections in this paper:

$$
\begin{aligned}
\widehat{\mathbf{H}}_{b}^{H} \widehat{\mathbf{H}}_{b} & =\widetilde{\mathbf{U}} \widetilde{\boldsymbol{\Lambda}} \widetilde{\mathbf{U}}^{H}, \quad \widetilde{\mathbf{U}}=\left[\begin{array}{lll}
\widetilde{\mathbf{u}}_{1} & \cdots & \widetilde{\mathbf{u}}_{n_{T}}
\end{array}\right], \quad \widetilde{\mathbf{U}}^{H} \widetilde{\mathbf{U}}=\mathbf{I} \\
\widetilde{\boldsymbol{\Lambda}} & =\operatorname{diag}\left(\widetilde{\lambda}_{1}, \cdots, \widetilde{\lambda}_{n_{T}}\right), \quad \widetilde{\lambda}_{1} \geq \cdots \geq \widetilde{\lambda}_{n_{T}} \geq 0 \\
\mathbf{B} & =\sqrt{P_{T}} \sum_{k=1}^{n_{T}} \sqrt{p_{k}} \widetilde{\mathbf{u}}_{k} \mathbf{t}_{k}^{H}=\sqrt{P_{T}} \widetilde{\mathbf{U}} \mathbf{P} \mathbf{T}^{H} \\
\mathbf{P} & =\operatorname{diag}\left(\sqrt{p_{1}}, \cdots, \sqrt{p_{n_{T}}}\right), \quad \mathbf{T}^{H} \mathbf{T}=\mathbf{I}
\end{aligned}
$$

This signal models consists in a transmission through the different eigenmodes of the estimated channel. This transmission is decoupled by means of using a set of $n_{T}$ orthonormal temporal signatures $\left\{\mathbf{t}_{k}\right\}_{k=1}^{n_{T}}\left(\mathbf{t}_{k} \in \mathbb{C}^{n_{T} \times 1}\right)$ which are collected in the unitary matrix $\mathbf{T}=\left[\begin{array}{lll}\mathbf{t}_{1} & \cdots & \mathbf{t}_{n_{T}}\end{array}\right]$. The powers $\left\{p_{k}\right\}_{k=1}^{n_{T}}$ are responsible for giving a relative importance to the different estimated eigenmodes. It can be easily shown that the power constraint $\|\mathbf{B}\|_{F}^{2}=P_{T}$ can be reformulated as $\sum_{k=1}^{n_{T}} p_{k}=1$. According to this signalling method, it can be shown that the SNR can be expressed as SNR $=P_{T} \frac{\sigma_{s}^{2}}{\sigma_{w}^{2}} \operatorname{tr}\left(\widetilde{\mathbf{U}}{ }^{H} \mathbf{H}^{H} \mathbf{H} \widetilde{\mathbf{U}} \mathbf{P}^{2}\right)$, which is independent of the unitary matrix $\mathbf{T}$.

As shown in this signal model, $n_{T}$ channel uses or periods of time are needed to transmit one symbol. Obviously, this implies a reduction of the useful signal rate by a factor $n_{T}$. If a full rate system is desired, then $n_{T}$ different symbols $\left\{s_{k}(n)\right\}_{k=1}^{n_{T}}$ have to be transmitted simultaneously, according to the following scheme:

$$
\mathbf{X}_{T}(n)=\sum_{k=1}^{n_{T}} \mathbf{B}_{k} s_{k}(n)=\sqrt{P_{T}} \sum_{k=1}^{n_{T}} \widetilde{\mathbf{U}} \mathbf{P} \mathbf{T}_{k}^{H} s_{k}(n)
$$

that is, the same importance is given to the estimated eigenmodes for all the symbols, but different temporal signatures are applied. Obviously, the problem consists in decoupling the detection of the symbols at the receiver without decreasing the SNR. For real constellations, this can be done by using the unitary matrices deduced for Orthogonal Space Time Block Coding (OSTBC) ([1] [2]). For the case of complex symbols, only a $1 / 2$ rate transmission can be achieved for any number of transmit antennas. In the following

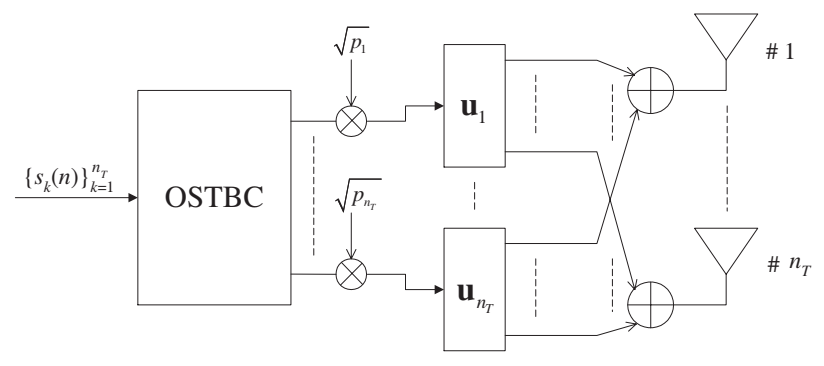

Fig. 1. Transmitter architecture combining OSTBC and weighted beamforming.

sections we assume BPSK symbols and all the analysis is carried out as if only one symbol is transmitted.

To summarize, the transmitter architecture proposed in this paper is based on the concatenation of an OSTBC and a set of $n_{T}$ beamformers (similarly to [9] and [10]), each one corresponding to an eigenmode of the estimated MIMO channel and applied to an output of the OSTBC. The set of powers $\left\{p_{k}\right\}_{k=1}^{n_{T}}$ are responsible for scaling the outputs of the OSTBC (see Fig. 1). In the following sections, it is shown how to design and calculate the set of powers according to different criteria, such as a maximin robust technique.

\section{NON-ROBUST BEAMFORMING}

In this section we focus the attention on the design of a transmitter in which it is assumed that the available channel estimate is perfect, that is, $\mathrm{SNR}_{\mathrm{est}}=\infty$ and $\mathbf{H}=\widehat{\mathbf{H}}=\widehat{\mathbf{H}}_{b}$, leading to a non-robust classical design. Taking this into account, the SNR during the design stage can be assumed to be equal to:

$$
\begin{aligned}
\mathrm{SNR} & =P_{T} \frac{\sigma_{s}^{2}}{\sigma_{w}^{2}} \operatorname{tr}\left(\widetilde{\mathbf{U}}^{H} \widehat{\mathbf{H}}_{b}^{H} \widehat{\mathbf{H}}_{b} \widetilde{\mathbf{U}} \mathbf{P}^{2}\right)=P_{T} \frac{\sigma_{s}^{2}}{\sigma_{w}^{2}} \operatorname{tr}\left(\widetilde{\Lambda} \mathbf{P}^{2}\right) \\
& =P_{T} \frac{\sigma_{s}^{2}}{\sigma_{w}^{2}} \sum_{k=1}^{n_{T}} \widetilde{\lambda}_{k} p_{k}
\end{aligned}
$$

According to this expression, the optimization problem can be formulated as follows:

$$
\begin{array}{rc}
\max _{\left\{p_{k}\right\}_{k=1}^{n}} & \sum_{k=1}^{n_{T}} \tilde{\lambda}_{k} p_{k} \\
\text { s.t. } & \sum_{k=1}^{n_{T}} p_{k}=1, \quad p_{k} \geq 0
\end{array}
$$

The solution of this constrained optimization corresponds to $p_{1}=1$ and $p_{k}=0, \forall k>1$, i.e., a transmission scheme in which only the first output of the OSTBC block is transmitted through the application of the maximum eigenmode $\widetilde{\mathbf{u}}_{1}$ of the MIMO channel estimate. This is equivalent to classical beamforming in which no space-time coding is applied, due to the fact that in conventional OSTBC the first output is equal to the original symbol stream.

\section{THE MAXIMIN ROBUST APPROACH}

In this section we propose a design of the power parameters $\left\{p_{k}\right\}$ under a maximin perspective [7]. This design criterion takes into account that there is some error in the available channel estimate at the transmitter. Due to the presence of the error, the performance 
in terms of SNR can decrease if the transmitter is designed according to the channel estimate without taking into account this error. A possible strategy that is less sensitive to this effect consists in applying a maximin approach in the design. In this design the objective is to look, given a power distribution, for the worst channel $\mathbf{H}^{*}$ within an uncertainty area $\mathcal{H}\left(\widehat{\mathbf{H}}_{b}\right)$ around the channel estimate $\widehat{\mathbf{H}}_{b}$, i.e., to look for the real channel that minimizes the SNR for a concrete power distribution. Once that this worst channel is found, the second step consists in maximizing the SNR for this worst channel by designing the power parameters adequately. Before presenting the design, let us show some useful definitions:

$$
\begin{aligned}
& M\left(\left\{p_{k}\right\}, \mathbf{H}\right)= \operatorname{tr}\left(\widetilde{\mathbf{U}}^{H} \mathbf{H}^{H} \mathbf{H} \widetilde{\mathbf{U}} \mathbf{P}^{2}\right) \\
& \mathbf{H}= \widehat{\mathbf{H}}_{b}+\boldsymbol{\Delta}, \quad\|\boldsymbol{\Delta}\|_{F}^{2} \leq \epsilon \\
& M\left(\left\{p_{k}\right\}, \mathbf{H}\right)= \operatorname{tr}\left(\widetilde{\mathbf{U}}^{H} \widehat{\mathbf{H}}_{b}^{H} \widehat{\mathbf{H}}_{b} \widetilde{\mathbf{U}} \mathbf{P}^{2}\right)+\operatorname{tr}\left(\widetilde{\mathbf{U}}^{H} \widehat{\mathbf{H}}_{b}^{H} \boldsymbol{\Delta} \widetilde{\mathbf{U}} \mathbf{P}^{2}\right)+ \\
& \operatorname{tr}\left(\widetilde{\mathbf{U}}^{H} \boldsymbol{\Delta}^{H} \widehat{\mathbf{H}}_{b} \widetilde{\mathbf{U}} \mathbf{P}^{2}\right)+\operatorname{tr}\left(\widetilde{\mathbf{U}}^{H} \boldsymbol{\Delta}^{H} \boldsymbol{\Delta} \widetilde{\mathbf{U}} \mathbf{P}^{2}\right)(10) \\
& \mathbf{h}=\operatorname{vec}(\mathbf{H}), \widehat{\mathbf{h}}_{b}=\operatorname{vec}\left(\widehat{\mathbf{H}}_{b}\right), \delta=\operatorname{vec}(\boldsymbol{\Delta}),\|\delta\|^{2}=\delta^{H} \delta \leq \epsilon \\
& \mathcal{H}\left(\widehat{\mathbf{H}}_{b}\right)=\left\{\mathbf{H}: \mathbf{H}=\widehat{\mathbf{H}}_{b}+\boldsymbol{\Delta},\|\boldsymbol{\Delta}\|_{F} \leq \sqrt{\epsilon}\right\}
\end{aligned}
$$

where in this case the uncertainty area $\mathcal{H}\left(\widehat{\mathbf{H}}_{b}\right)$ is represented by the Euclidean norm represented by $\|\Delta\|_{F}$ and $\|\delta\|$, corresponding to a sphere centered at the Bayesian channel estimate $\widehat{\mathbf{H}}_{b}$ and a radius equal to $\sqrt{\epsilon}$, and the cost function $M(\cdot)$ is proportional to the SNR. According to this, the maximin optimization and design problem for a concrete channel estimate can be formulated as:

$$
\max _{\left\{p_{k}\right\}} \min _{\mathbf{H} \in \mathcal{H}\left(\widehat{\mathbf{H}}_{b}\right)} M\left(\left\{p_{k}\right\}, \mathbf{H}\right)
$$

In (10) there is a quadratic term on the error in the channel estimate $\delta$. For usual values of $\mathrm{SNR}_{\mathrm{est}}$ this term is negligible, and therefore, $M(\cdot)$ can be simplified as (note that the first term in (10) has been rewritten in terms of the eigenvalues $\left\{\widetilde{\lambda}_{k}\right\}$ of $\widehat{\mathbf{H}}_{b}^{H} \widehat{\mathbf{H}}_{b}$ ):

$$
\widetilde{M}=\sum_{k=1}^{n_{T}} \widetilde{\lambda}_{k} p_{k}+\operatorname{tr}\left(\widetilde{\mathbf{U}}^{H} \widehat{\mathbf{H}}_{b}^{H} \boldsymbol{\Delta} \widetilde{\mathbf{U}} \mathbf{P}^{2}\right)+\operatorname{tr}\left(\widetilde{\mathbf{U}}^{H} \boldsymbol{\Delta}^{H} \widehat{\mathbf{H}}_{b} \widetilde{\mathbf{U}}^{2}\right)
$$

The component $\operatorname{tr}\left(\widetilde{\mathbf{U}}^{H} \widehat{\mathbf{H}}_{b}^{H} \boldsymbol{\Delta} \widetilde{\mathbf{U}} \mathbf{P}^{2}\right)$ can be simplified using the equality $\operatorname{tr}(\mathbf{A B C D})=\operatorname{vec}\left(\mathbf{D}^{H}\right)^{H}\left(\mathbf{C}^{T} \otimes \mathbf{A}\right) \operatorname{vec}(B)$ :

$$
\begin{aligned}
\operatorname{tr}\left(\widetilde{\mathbf{U}}^{H} \widehat{\mathbf{H}}_{b}^{H} \boldsymbol{\Delta} \widetilde{\mathbf{U}} \mathbf{P}^{2}\right) & =\mathbf{p}^{T} \mathbf{A}^{H} \delta, \quad \mathbf{A}^{H} \in \mathbb{C}^{n_{T} \times n_{T} n_{R}} \\
\widetilde{M}\left(\left\{p_{k}\right\}, \delta\right) & =\sum_{k=1}^{n_{T}} \widetilde{\lambda}_{k} p_{k}+\mathbf{p}^{T} \mathbf{A}^{H} \delta+\delta^{H} \mathbf{A p}
\end{aligned}
$$

where the matrix $\mathbf{A}^{H}$ is constructed by choosing only the rows $1+k\left(1+n_{T}\right), \quad k=0, \ldots, n_{T}-1$ of $\widetilde{\mathbf{U}}^{T} \otimes\left(\widetilde{\mathbf{U}}^{H} \widehat{\mathbf{H}}_{b}^{H}\right)$, and $\mathbf{p}=\left[\begin{array}{lll}p_{1} & \cdots & p_{n_{T}}\end{array}\right]^{T}$. The first step to find the optimum power parameters consists in finding the channel in the uncertainty area $\mathcal{H}\left(\widehat{\mathbf{H}}_{b}\right)$ or, equivalently, the vector $\delta$ such that $\|\delta\|^{2} \leq \epsilon$, that minimizes the modified cost function $\widetilde{M}(\cdot)$. As the modified cost function depends linearly on $\delta$, it is easy to verify that the minimization is achieved when $\|\delta\|^{2}=\epsilon$. Finally, the vector $\delta$ minimizing $\widetilde{M}(\cdot)$ subject to the norm constraint is $\delta^{*}=-\sqrt{\epsilon} \frac{\mathbf{A p}}{\|\mathbf{A} \mathbf{p}\|}$.
According to this result, the function to be maximized with respect to the power parameters $\left\{p_{k}\right\}$ is:

$$
\begin{aligned}
\max _{\left\{p_{k}\right\}_{k=1}^{n}} & \sum_{k=1}^{n_{T}} \widetilde{\lambda}_{k} p_{k}-2 \sqrt{\epsilon}\|\mathbf{A} \mathbf{p}\| \\
\text { s.t. } & \sum_{k=1}^{n_{T}} p_{k}=1, \quad p_{k} \geq 0
\end{aligned}
$$

This is a convex optimization problem that can be solved using iterative and powerful algorithms such as the "interior point method" [11]. Once the optimum powers $\left\{p_{k}^{*}\right\}$ have been found, the designer can calculate which is the required transmit power so as to guarantee the desired $\mathrm{SNR}_{t}$ for any channel in the uncertainty area as: $P_{T}^{*}=\mathrm{SNR}_{t} \frac{\sigma_{w}^{2}}{\sigma_{s}^{2} M\left(\left\{p_{k}^{*}\right\}, \delta^{*}\right)}$ (i.e., $\mathrm{SNR}_{t}$ represents the minimum Quality of Service (QoS) desired by the user).

\subsection{Uncertainty Area}

Up to this point it has been assumed that the parameter $\epsilon$ is known. In this subsection two possibilities are proposed to calculate the size of the uncertainty area.

\subsubsection{Outage Probability}

As it has been shown in Sec. 2, the error $\delta=\mathbf{h}-\widehat{\mathbf{h}}_{b}$ in the Bayesian channel estimate $\widehat{\mathbf{h}}_{b}$ is a white properly Gaussian distributed vector with zero mean and covariance $\mathbf{C}=\frac{\sigma_{h}^{2}}{1+\mathrm{SNR}_{\mathrm{est}}} \mathbf{I}$. Consequently, $\|\delta\|^{2}$ follows a chi-square distribution with $2 n_{R} n_{T}$ degrees of freedom and normalized variance $\frac{\sigma_{h}^{2}}{2\left(1+\mathrm{SNR}_{\mathrm{est}}\right)}$. By means of this, a minimum quality can be guaranteed to the user or communication by relating the outage probability $P_{\text {out }}$ of having a SNR higher than or equal to the desired one $\mathrm{SNR}_{t}$, and the size of the uncertainty area as $\epsilon=f^{-1}\left(P_{\text {out }}\right)$, where $f(\cdot)$ is the Cumulative Density Function (CDF) of the chi-square distribution.

The main problem of this technique is that for high outage probabilities, the errors in the channel estimate may be too high so as to consider the first order approximation of the cost function $M(\cdot)$ (see eq. (14)). Just to clarify this idea, if $\epsilon \geq\left\|\widehat{\mathbf{h}}_{b}\right\|^{2}$, then the worst channel in the unvertainty area would be $\mathbf{h}^{*}=\mathbf{0}$, i.e. $\delta^{*}=-\widehat{\mathbf{h}}_{b}$, however the first order approximation would state that the worst channel would correspond to $\delta^{*}=-\sqrt{\epsilon} \frac{\mathbf{A p}}{\|\mathbf{A p}\|}$. In this case it is quite difficult to guarantee a minimum SNR to the user; consequently, a more reasonable option would consist in not transmitting any symbol, instead of increasing the transmit power.

\subsubsection{Adhoc Approach}

In order to solve the problem of having very large uncertainty areas that contain $\mathbf{h}^{*}=\mathbf{0}$ as the worst channel, it is possible to calculate the parameter $\epsilon$ without having any relationship with the outage probability. In this case, we propose the following adhoc rule: $\epsilon=\frac{1}{1+\mathrm{SNR}_{\mathrm{est}}}\left\|\widehat{\mathbf{h}}_{b}\right\|^{2}$. In this design, the channel $\mathbf{h}=\mathbf{0}$ does not belong to the uncertainty area. Besides, as $\mathrm{SNR}_{\text {est }}$ increases, the size of the uncertainty area decreases, as expected. It can be shown that, usually, for this kind of uncertainty areas, the first order approximation presented in eq. (14) is quite accurate.

\section{SIMULATION RESULTS AND CONCLUSIONS}

In this section we present several results corresponding to the application of the already presented robust design, comparing the 


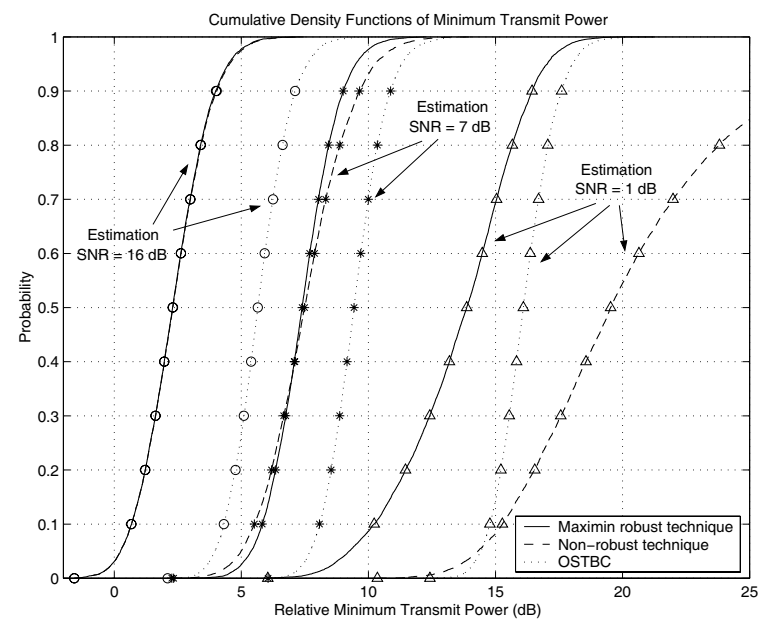

Fig. 2. Cumulative density functions for the minimum transmit power for $\mathrm{SNR}_{t}=10 \mathrm{~dB}$. Techniques: robust maximin design (adhoc approach), non-robust beamforming and OSTBC

results with other classical techniques such as OSTBC and the non-robust beamforming approach. In all cases, the objective is to evaluate which is the minimum transmit power to guarantee a minimum QoS in terms of a target $\mathrm{SNR}_{t}$, that is, in the simulations it is calculated how much power is necessary at the transmitter so that the communication has a SNR higher than or equal to $\mathrm{SNR}_{t}$ for any channel realization within an uncertainty area around the channel estimate. In all the simulations it is assumed that $\mathrm{SNR}_{t}=10 \mathrm{~dB}$, $\sigma_{h}^{2}=1$, and that the signal and noise power are $\sigma_{s}^{2}=\sigma_{w}^{2}=1$.

In Fig. 2 the CDF's of the minimum transmit power required to guarantee $\mathrm{SNR} \geq \mathrm{SNR}_{t}$ for any channel in the uncertainty area around the estimate are presented. The considered techniques are the maximin robust design using the adhoc uncertainty area presented in 4.1.2, the non-robust beamforming and the OTSBC approaches for a system with $n_{T}=n_{R}=4$ antennas. As it can be seen, the minimum required transmit power for the robust design is lower than the other techniques as the estimation SNR decreases, as expected. Besides, for very low estimation SNR the OSTBC solution may need less power than the non-robust approach.

In Fig. 3 it is represented the mean value of the minimum transmit power to obtain a minimum QoS, as specified in the previous figure. In this case, the outage probability approach has been taken for two different antennas configurations: $n_{T}=n_{R}=4$ and $n_{T}=n_{R}=8$, and two values of the outage probability: 0.7 and 0.9 . As expected, for higher values of the outage probability and less number of antennas, more transmit power is necessary. Besides, as the estimation SNR decreases, the difference between the necessary transmit power for the robust and the non-robust approaches increases, as expected.

\section{REFERENCES}

[1] V. Tarokh, H. Jafharkani, and A. R. Calderbank, "SpaceTime Block Codes from Orthogonal Designs," IEEE Trans. Inform. Theory, vol. 45, no. 5, pp. 1456-1467, July 1999.

[2] G. Ganesan and P. Stoica, "Space-Time Block Codes: A

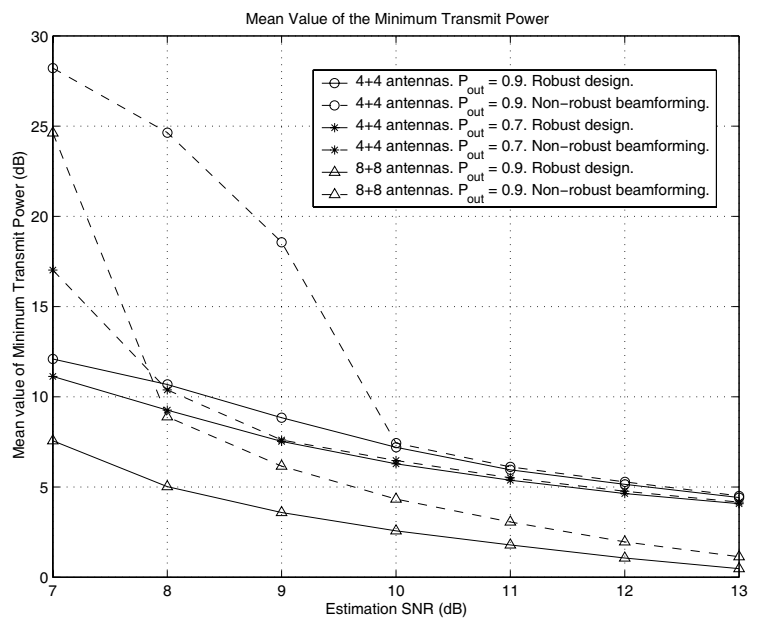

Fig. 3. Mean value of the minimum transmit power for $\mathrm{SNR}_{t}=10$ $\mathrm{dB}$. Techniques: robust maximin design (outage probability approach) and non-robust beamforming.

Maximum SNR Approach," IEEE Trans. Inform. Theory, vol. 47, no. 4, pp. 1650-1656, May 2001.

[3] A. Pascual-Iserte, A. I. Pérez-Neira, and M. A. Lagunas, "Joint Beamforming Strategies in OFDM-MIMO Systems," in Proc. IEEE Int. Conf. Acoust., Speech, Signal Proc., ICASSP'02, May 2002, vol. 3, pp. 2845-2848.

[4] A. Pascual-Iserte, A. I. Pérez-Neira, and M. A. Lagunas, "On Power Allocation Strategies for Maximum Signal to Noise and Interference Ratio in an OFDM-MIMO System," accepted at IEEE Trans. on Wireless Comm., April 2003.

[5] F. Rey, M. Lamarca, and G. Vázquez, "Optimal Power Allocation with Partial Channel Knowledge for MIMO Multicarrier Systems," in Proc. IEEE 56th Vehicular Technology Conference, VTC'02, Sept. 2002, vol. 4, pp. 2121-2125.

[6] A. Pascual-Iserte, A. I. Pérez-Neira, and M. A. Lagunas, "Exploiting Transmission Spatial Diversity in Frequency Selective Systems with Feedback Channel," in Proc. IEEE Int. Conf. Acoust., Speech, Signal Proc., ICASSP'03, April 2003.

[7] S. A. Kassam and H. V. Poor, "Robust Techniques for Signal Processing: A Survey," Proceedings of the IEEE, vol. 73, no. 3, pp. 433-481, March 1985.

[8] S. A. Vorobyov, A. B. Gershman, and Z-Q. Luo, "Robust Adaptive Beamforming Using Worst-Case Performance Optimization: A Solution to the Signal Mismatch Problem," IEEE Trans. Signal Proc., vol. 51, pp. 313-324, Febr. 2003.

[9] G. Jöngren, M. Skoglund, and B. Ottersten, "Combining Beamforming and Orthogonal Space-Time Block Coding," IEEE Trans. Inform. Theory, vol. 48, no. 3, pp. 611-627, March 2002.

[10] S. Zhou and G. B. Giannakis, "Adaptive Modulation for Multi-Antenna Transmissions with Channel Mean Feedback," in Proc. IEEE Int. Conf. Comm., ICC'03, May 2003.

[11] S. Boyd and L. Vandenberghe, Introduction to Convex Optimization with Engineering Applications, Course Notes. Stanford University, 2000. 